\title{
Calidad de atención programa AUGE cáncer cérvicoute- rino: diferencias y similitudes entre usuarias y profesionales
}

\author{
Miriam Ximena Concha P. MSc ${ }^{a}$, María Teresa Urrutia S. $P h D^{a}$. \\ a Enfermera Matrona, Departamento de Salud de la Mujer, Escuela de Enfermería, Facultad de Medicina, Pontificia \\ Universidad Católica de Chile.
}

Proyecto financiado por el Fondo Nacional de Investigación en Salud, CONICYT-MINSAL, CHILE. Proyecto FONIS SA05I20047.

\section{RESUMEN}

Antecedentes: El cáncer cérvicouterino (CC) es considerada patología GES. Desde su implementación no se reporta evaluación de la calidad de atención del Programa. Objetivo: Conocer la percepción de la calidad de la atención recibida y otorgada en el Programa AUGE-CC del Servicio de Salud Metropolitano Sur Oriente. Método: Estudio descriptivo de corte transversal con un grupo de 364 usuarias y 59 profesionales de los tres niveles de atención. Para evaluar la calidad de atención de usuarias y profesionales se utilizó el instrumento INCA-AUGE. Resultados: Dimensión I: La nota brindada al programa por las usuarias fue 6,3 versus 5,9 de los profesionales. Dimensión II: $78 \%$ de las usuarias percibe un trato humanizado, versus el $48 \%$ de los profesionales que perciben otorgar un trato humanizado en la atención. Dimensión III: $40 \%$ de las mujeres considera suficiente la dotación de personal del programa, a diferencia de los profesionales en que solo el 15,3\% lo considera adecuado. Dimensión IV: 93\% del grupo de mujeres perciben recibir educación sobre su enfermedad y tratamiento versus el $64,4 \%$ de los profesionales que lo considera. Dimensión V: $80 \%$ de las usuarias percibe que el programa cuenta con los materiales adecuados para entregar una buena atención, a diferencia del $5,1 \%$ de los profesionales que los consideran adecuados. Conclusión: La evaluación en general es buena siendo los profesionales más exigentes. Se identifican áreas de mejora emergentes a través de la comparación de percepciones de usuarias y profesionales, figuras claves implicadas en la evaluación de la calidad de atención.

\section{PALABRAS CLAVE: Cáncer cérvicouterino, calidad de atención, programa AUGE, evaluación de programas en salud}

\section{SUMMARY}

Background: Cervical Cancer (CC) is a GES pathology. Since its implementation, evaluation of the program's quality of care have not been reported. Objective: Determine the perception of the quality of care received and given in the AUGE Program CC of the Servicio de Salud Metropolitano Sur Oriente. Methodology: Descriptive cross sectional study with a group of 364 users and 59 professionals from the three levels of care. To assess the quality of care of users and professionals was used the instrument INCA-AUGE was used. Results: Dimension I: The grade given by users to this program was a 6.3 versus a 5.9 given by professionals. Dimension II: $78 \%$ of users perceive a humane treatment, versus a $48 \%$ of professionals which perceive that treatment provided is humane. Dimension III: $40 \%$ of women consider that the amount of people working in the program is adequate, unlike professionals that only a $15.3 \%$ considers it adequate. Dimension IV: $93 \%$ of the group of women perceives to be educated about their disease and treatment versus $64.4 \%$ of 
professionals considers it. Dimension V: $80 \%$ of users perceived that the program has adequate materials to provide a good care, versus a $5.1 \%$ of professionals who consider them appropriate. Conclusion: The evaluation is generally good being professionals more demanding. There have been identified emerging areas for improvement by comparing the perceptions of users and professionals, key figures involved in the evaluation of the quality of care.

\section{KEY WORDS: Cervical cancer, quality of care, AUGE program, health program evaluation}

\section{INTRODUCCIÓN}

Uno de los objetivos sanitarios planteados en Chile para la década 2000-2010, fue responder a las expectativas de la población respecto al sistema de salud (1), determinando con ello la necesidad de evaluar la Calidad de Atención (CA), entendiendo por ésta aquellos estándares que permiten a las instituciones acreditadas y especialistas certificados, entregar una adecuada y correcta atención ajustada a las demandas de los usuarios de los servicios sanitarios $(2,3)$.

La Reforma de Salud chilena establece el "Régimen General de Garantías Explicitas en Salud" (GES), diseñado para un grupo prioritario de patologías debido al alto costo social que conllevan estas enfermedades, siendo el cáncer cérvicouterino (CC) una de ellas $(2,4)$. Hasta la fecha, si bien existe literatura que evalúe de forma general las GES (5), no existe literatura que reporte evaluación de la CA otorgada por los programas, menos si se intenta evaluar desde la perspectiva de los profesionales (6-11), actores importantes a la hora de tomar decisiones en salud e introducir mejoras en la atención $(12,13,14)$.

El objetivo de esta investigación es conocer la percepción sobre la CA recibida por las usuarias y otorgada por los profesionales del Programa AUGE-CC del Servicio de Salud Metropolitano Sur Oriente (SSMSO).

\section{MATERIAL Y METODO}

Estudio descriptivo de corte transversal realizado en un grupo de usuarias del programa AUGECC del SSMSO y los profesionales que las atienden. Del universo de 852 mujeres, se seleccionó una muestra aleatoria con un $95 \%$ de confianza la que contempló 237 mujeres con lesiones preinvasoras (LIE) y 127 mujeres con CC, dando un total de 364 usuarias. Los criterios de inclusión para las usuarias fueron haber ingresado al programa AUGE-CC entre el 1ํ de diciembre 2004 y el 31 de diciembre 2005, diagnóstico histopatológico de LIE o CC y haber recibido atención y tratamiento en el SSMSO. Las mujeres fueron ingresadas al estudio entre mayo y octubre de 2006, fueron entrevistadas en sus domicilios por enfermeras matronas y matronas previamente entrenadas. Las variables sociodemográficas y médicas se obtuvieron de las fichas clínicas respectivas. La percepción de las usuarias sobre la CA se midió utilizando el instrumento INCA-AUGE (Instrumento Calidad de Atención- AUGE) (9) que tiene 19 ítems distribuidos en 5 dimensiones:

Dimensión I: funcionamiento del programa (6 ítems) Dimensión II: relación usuaria-profesional (6 ítems) Dimensión III: infraestructura y recurso humano (3 ítems)

Dimensión IV: educación sobre la enfermedad (2 ítems)

Dimensión V: recursos materiales (2 Ítems).

La dimensión de Funcionamiento del Programa se evalúa con dos escalas diferentes. Una de las escala es numérica de 1 a 7 donde 1 es muy malo y 7 excelente, la otra corresponde a una escala de Likert 5 (siempre, casi siempre, a veces, casi nunca y nunca). Las cuatro dimensiones restantes son evaluadas con escala Likert 3 ( $\mathrm{Si}$, Parcialmente y No). EI INCA-AUGE es un instrumento desarrollado y validado en población chilena, y en la presente muestra su confiabilidad medida por Alfa de Crombach fue de 0,70.

En relación a los profesionales $(n=59)$, contempló a la totalidad de los profesionales involucrados en el programa AUGE-CC en los diferentes niveles de atención del SSMSO, incluyendo matronas de los tres niveles, médicos ginecólogos del nivel secundario y terciario, y médicos radiólogos, más una enfermera del Instituto Nacional del Cáncer (INC) donde las usuarias reciben tratamiento de radioterapia. Para esta investigación, a nivel de la atención primaria, sólo se consideró al profesional responsable del programa. Los profesionales incluidos corresponden a todos aquellos que tienen una relación directa con las usuarias en los diferentes 
niveles de atención con una permanencia en el cargo superior a seis meses.

Para la obtención de la información referida a la percepción de la CA de los profesionales, se aplicó el instrumento INCA-AUGE descrito anteriormente y modificado para profesionales. La recolección de la información fue realizada entre los meses de agosto 2006 y enero 2007 en cada uno de los centros de atención. Los comités de ética de la Escuela de Enfermería de la Pontificia Universidad Católica de Chile y del SSMSO aprobaron esta investigación. Usuarias y profesionales, firmaron un consentimiento informado. El análisis de los datos se realizó por medio del programa estadístico SPSS 17.0. Se realizaron medidas de tendencia central, de dispersión y análisis de frecuencia.

\section{RESULTADOS}

El promedio de edad de las mujeres fue de 42,2 $\pm 11,9$ años. La escolaridad promedio correspondió a 9,03 años $\pm 3,5$ años. Un 3,3\% tenía educación superior (Tabla I). La edad promedio de los profesionales fue de 39,15 $\pm 10,26$ años (rango: 25 a 70 años). De los 59 profesionales 45 son matronas(es), 13 médicos y 1 enfermera. El 69,5\% trabaja en el nivel primario, el $6,8 \%$ en el nivel secundario, el $20,4 \%$ en el nivel terciario, y el $3,4 \%$ en el nivel secundario y terciario. Cabe señalar que en el nivel primario se desempeñan solo matronas(es), en el nivel secundario matronas(es) y médicos ginecólogos y en el nivel terciario matronas(es), médicos ginecólogos, radiólogos y una enfermera. El tiempo promedio en el cargo corresponde a 7,7 \pm 7,4 años (rango: 6 meses a 34 años). La totalidad de los profesionales médicos refiere tener especialización en oncología.

La evaluación de la CA, respecto de la Dimensión I (Funcionamiento del programa), que entregan los profesionales al programa en general, es menor que la entregada por las usuarias, sin embargo, en la evaluación que hacen del equipo de profesiona- les, la evaluación realizada por ellos es mejor que el que hacen las mujeres $(6,7$ y 6,2 respectivamente) (Tabla II). Al analizar los resultados de los otros ítems de esta dimensión, los que presentan mayores discrepancias entre usuarias y profesionales, es la calidad de comunicación establecida, en donde, las mujeres evalúan este ítem como muy buena o buena en un $85,5 \%$ versus el $49,2 \%$ de los profesionales. El otro ítem que es importante señalar en cuanto a sus diferencias es el de la capacidad del programa para solucionar problemas productos del $\mathrm{CC}$, el $93 \%$ de las usuarias lo percibe en forma satisfactoria, versus el $67,8 \%$ de los profesionales. Un punto de acuerdo entre usuarias y profesionales es la recomendación a otra persona, familiar o amiga con esta enfermedad atenderse en este programa (Tabla III).

Tabla I

\section{CARACTERÍSTICAS DE LAS USUARIAS PARTICIPANTES DEL ESTUDIO}

\begin{tabular}{llcr}
\hline Variable & & $\mathrm{n}$ & $\%$ \\
\hline Previsión salud & FONASA A & 188 & 51,6 \\
& FONASA B & 100 & 27,5 \\
& FONASA C & 47 & 12,9 \\
& FONASA D & 24 & 6,6 \\
& PRAIS & 4 & 1,1 \\
Último nivel & No sabe & 1 & 0,1 \\
de escolaridad & Analfabeta & 8 & 2,2 \\
aprobado & Básica & 140 & 38,5 \\
& Media & 191 & 52,5 \\
& Técnica & 12 & 3,3 \\
& Universitaria & 11 & 3,0 \\
\hline
\end{tabular}

FONASA A: carentes de recursos o indigentes. FONASA $\mathrm{B}$ : ingreso imponible mensual $\leq$ a $\$ 182.000$. FONASA C: Ingreso imponible mensual > a $\$ 182.000 \mathrm{y} \leq \mathrm{a} \$ 265.000$. FONASA D: Ingreso imponible mensual > a $\$ 265.000$ (vigente desde el 14 de julio de 2011). 1US\$: $\$ 463,65$ al 14 de julio de 2011. PRAIS: Programa de Reparación y Atención Integral de Salud.

Tabla II

\section{DIMENSIÓN I: FUNCIONAMIENTO DEL PROGRAMA. EVALUACIÓN DEL PROGRAMA Y DE LOS PROFESIONALES}

\begin{tabular}{lcccc}
\hline \multicolumn{1}{c}{ Funcionamiento del Programa } & \multicolumn{2}{c}{ Calificación } & \multicolumn{2}{c}{ Rango } \\
\multicolumn{1}{c}{ CC-AUGE } & $\begin{array}{c}\text { Usuaria } \\
(\mathrm{n}=352)\end{array}$ & $\begin{array}{c}\text { Profesional } \\
(\mathrm{n}=59)\end{array}$ & $\begin{array}{c}\text { Usuaria } \\
(\mathrm{n}=352)\end{array}$ & $\begin{array}{c}\text { Profesional } \\
(\mathrm{n}=59)\end{array}$ \\
\hline $\begin{array}{l}\text { Calificación asignada a los profesionales } \\
\text { del programa }\end{array}$ & $6,2 \pm 0,8$ & $6,7 \pm 0,3$ & $2-7$ & $2-7$ \\
\begin{tabular}{l} 
Calificación asignada al programa \\
\hline
\end{tabular} & $6,3 \pm 5,9$ & $5.9 \pm 0,5$ & $1-7$ & $2-7$ \\
\hline
\end{tabular}




\section{Tabla III}

\section{DIMENSIÓN I: FUNCIONAMIENTO DEL PROGRAMA}

\begin{tabular}{|c|c|c|c|c|c|c|}
\hline & $\begin{array}{l}\text { Usuaria } \\
(\mathrm{n}=352)\end{array}$ & $\begin{array}{l}\text { Profesional } \\
(n=59)\end{array}$ & $\begin{array}{l}\text { Usuaria } \\
(\mathrm{n}=352)\end{array}$ & $\begin{array}{l}\text { Profesional } \\
\quad(n=59)\end{array}$ & $\begin{array}{l}\text { Usuaria } \\
(\mathrm{n}=352)\end{array}$ & $\begin{array}{c}\text { Profesional } \\
(\mathrm{n}=59)\end{array}$ \\
\hline & \multicolumn{2}{|c|}{ Siempre-Casi Siempre } & \multicolumn{2}{|c|}{ A veces } & \multicolumn{2}{|c|}{ Casi nunca-Nunca } \\
\hline \multirow{2}{*}{$\begin{array}{l}\text { Evaluación sobre la capacidad } \\
\text { de solucionar los problemas } \\
\text { productos de la enfermedad }\end{array}$} & $93,4 \%$ & $67,8 \%$ & $4,7 \%$ & $8,5 \%$ & $1,9 \%$ & $23,7 \%$ \\
\hline & \multicolumn{2}{|c|}{ Muy buena - Buena } & \multicolumn{2}{|c|}{ Regular } & \multicolumn{2}{|c|}{ Mala-Muy Mala } \\
\hline \multirow{2}{*}{$\begin{array}{l}\text { Evaluación de la calidad de } \\
\text { comunicación establecida con } \\
\text { la usuaria }\end{array}$} & $85,5 \%$ & $49,2 \%$ & $13,5 \%$ & $15,3 \%$ & $1,1 \%$ & $33.9 \%$ \\
\hline & \multicolumn{2}{|c|}{ Si } & \multicolumn{2}{|c|}{ Parcialmente } & \multicolumn{2}{|c|}{ No } \\
\hline \multirow{2}{*}{$\begin{array}{l}\text { Recomendación a otra perso- } \\
\text { na, familiar o amiga con esta } \\
\text { enfermedad para atenderse en } \\
\text { este programa }\end{array}$} & $93,7 \%$ & $86,4 \%$ & $3,9 \%$ & $6,8 \%$ & $1,9 \%$ & $6,8 \%$ \\
\hline & \multicolumn{2}{|c|}{$\mathrm{Si}$} & \multicolumn{2}{|c|}{ Parcialmente } & \multicolumn{2}{|c|}{ No } \\
\hline $\begin{array}{l}\text { Evaluación de la confianza } \\
\text { brindada }\end{array}$ & $79,1 \%$ & $55,9 \%$ & $19,5 \%$ & $23,7 \%$ & $1,1 \%$ & $20,3 \%$ \\
\hline
\end{tabular}

La Dimensión II (Relación usuaria profesional), es posible observar que existen diferencias importantes entre profesionales y usuarias. En cada uno de los ítems son los profesionales más exigentes al momento de la evaluación, llegando en algunos ítems a triplicar los valores (Tabla IV).

\section{DIMENSIÓN II: RELACIÓN USUARIA PROFESIONAL}

\begin{tabular}{|c|c|c|c|c|}
\hline & \multicolumn{2}{|c|}{ SI } & \multicolumn{2}{|c|}{ NO } \\
\hline & $\begin{array}{l}\text { Usuaria } \\
(\mathrm{n}=352)\end{array}$ & $\begin{array}{l}\text { Profesional } \\
\qquad(\mathrm{n}=59)\end{array}$ & $\begin{array}{l}\text { Usuaria } \\
(\mathrm{n}=352)\end{array}$ & $\begin{array}{l}\text { Profesional } \\
\quad(n=59)\end{array}$ \\
\hline Los profesionales se identifican por su nombre & $54,7 \%$ & $11,9 \%$ & $45,3 \%$ & $88,1 \%$ \\
\hline $\begin{array}{l}\text { Los profesionales se dan el tiempo para asegurarse que } \\
\text { las usuarias entienden la información entregada }\end{array}$ & $67 \%$ & $35,6 \%$ & $33 \%$ & $64,4 \%$ \\
\hline $\begin{array}{l}\text { Los profesionales dan opción de preguntar o explicar } \\
\text { inquietudes }\end{array}$ & $70,9 \%$ & $27,1 \%$ & $29,1 \%$ & $72,9 \%$ \\
\hline A las usuarias se les nombra por su nombre & $89 \%$ & $27,6 \%$ & $11 \%$ & $72,4 \%$ \\
\hline Las usuarias perciben un cuidado humanizado & $78 \%$ & $42,4 \%$ & $22 \%$ & $57,6 \%$ \\
\hline $\begin{array}{l}\text { A las usuarias, se les explica cómo y cuándo acudir a un } \\
\text { determinado lugar de la red }\end{array}$ & $85 \%$ & $37,3 \%$ & $15 \%$ & $62,7 \%$ \\
\hline
\end{tabular}


La Dimensión III (Infraestructura y recurso humano), es evaluada de manera similar por ambos grupos en lo referente a la infraestructura (57\% $42 \%$ ), sin embargo, no ocurre lo mismo al evaluar los espacios de espera y la cantidad de personal del programa, en donde nuevamente son los profesionales quienes evalúan en forma deficiente el programa (Tabla V).

La Dimensión IV (Educación sobre la patología), nuevamente es posible identificar diferencias porcentuales de casi 30 puntos entre los grupos, siendo nuevamente los profesionales más estrictos en sus juicios (Tabla $\mathrm{VI}$ ).

La Dimensión V (Recursos materiales), las diferencias son evidentes e igualmente son los profesionales los más exigentes. Un ítem a destacar es si el programa cuenta con los materiales adecuados para brindar una buena atención, en donde, el grupo de mujeres responde afirmativamente en un $80 \%$ a diferencia de los profesionales en que solo el $5,1 \%$ los percibe como adecuados (Tabla VII).

Tabla V

DIMENSIÓN III: INFRAESTRUCTURA Y RECURSO HUMANO

\begin{tabular}{|c|c|c|c|c|}
\hline & \multicolumn{2}{|c|}{$\mathrm{SI}$} & \multicolumn{2}{|c|}{ NO } \\
\hline & $\begin{array}{l}\text { Usuaria } \\
(\mathrm{n}=352)\end{array}$ & $\begin{array}{l}\text { Profesional } \\
\quad(n=59)\end{array}$ & $\begin{array}{l}\text { Usuaria } \\
(\mathrm{n}=352)\end{array}$ & $\begin{array}{l}\text { Profesiona } \\
\quad(\mathrm{n}=59)\end{array}$ \\
\hline $\begin{array}{l}\text { El Programa cuenta con una infraestructura } \\
\text { adecuada para otorgar una buena atención }\end{array}$ & $57 \%$ & $42,4 \%$ & $43 \%$ & $57,6 \%$ \\
\hline $\begin{array}{l}\text { Los espacios de espera para la atención } \\
\text { son cómodos }\end{array}$ & $53 \%$ & $25,4 \%$ & $47 \%$ & $74,6 \%$ \\
\hline $\begin{array}{l}\text { La dotación de personal es suficiente para } \\
\text { el buen funcionamiento para el programa }\end{array}$ & $40 \%$ & $15,3 \%$ & $60 \%$ & $84,7 \%$ \\
\hline
\end{tabular}

Tabla VI

DIMENSIÓN IV: EDUCACIÓN SOBRE LA ENFERMEDAD

\begin{tabular}{lcccccc}
\hline & $\begin{array}{c}\text { Usuarias } \\
(\mathrm{n}=352)\end{array}$ & $\begin{array}{c}\text { Profesionales } \\
(\mathrm{n}=59)\end{array}$ & $\begin{array}{c}\text { Usuarias } \\
(\mathrm{n}=352)\end{array}$ & $\begin{array}{c}\text { Profesionales } \\
(\mathrm{n}=59)\end{array}$ & $\begin{array}{c}\text { Usuarias } \\
(\mathrm{n}=352)\end{array}$ & $\begin{array}{c}\text { Profesionales } \\
(\mathrm{n}=59)\end{array}$ \\
\hline $\begin{array}{l}\text { Las usuarias reciben } \\
\text { educación sobre su } \\
\text { diagnóstico }\end{array}$ & $92,6 \%$ & $67,8 \%$ & $4,4 \%$ & $15,3 \%$ & $3 \%$ & $16,9 \%$ \\
$\begin{array}{l}\text { Las usuarias reciben } \\
\text { educación sobre su } \\
\text { tratamiento }\end{array}$ & $93,1 \%$ & $64,4 \%$ & $4,7 \%$ & $16,9 \%$ & $1,9 \%$ & $16,9 \%$ \\
\hline
\end{tabular}

Tabla VII

DIMENSIÓN V: RECURSOS MATERIALES

\begin{tabular}{|c|c|c|c|c|}
\hline & \multicolumn{2}{|c|}{ SI } & \multicolumn{2}{|c|}{ NO } \\
\hline & $\begin{array}{l}\text { Usuaria } \\
(n=352)\end{array}$ & $\begin{array}{l}\text { Profesional } \\
\quad(n=59)\end{array}$ & $\begin{array}{l}\text { Usuaria } \\
(n=352)\end{array}$ & $\begin{array}{l}\text { Profesional } \\
\quad(n=59)\end{array}$ \\
\hline $\begin{array}{l}\text { El Programa cuenta con los materiales } \\
\text { adecuados para otorgar una buena aten- } \\
\text { ción }\end{array}$ & $80 \%$ & $5,1 \%$ & $20 \%$ & $94,9 \%$ \\
\hline $\begin{array}{l}\text { El material utilizado se encuentra en buen } \\
\text { estado para otorgar una buena atención }\end{array}$ & $86 \%$ & $27,1 \%$ & $14 \%$ & $72,9 \%$ \\
\hline
\end{tabular}




\section{DISCUSIÓN}

A pesar de que el sector salud habitualmente realiza encuestas para evaluar la CA, éstas no suelen ser analizadas ni publicadas para introducir mejoras en los servicios sanitarios (11). La falta de estudios publicados dificulta la posibilidad de comparar los resultados de este estudio con otras realidades nacionales e internacionales.

De manera general en los diferentes ítems y dimensiones se encuentran diferencias en las percepciones entre usuarias y profesionales. Particularmente el grupo de los profesionales del Programa son más exigentes, lo que concuerda con otras investigaciones, donde ésta evaluación menos crítica por parte de los usuarios puede ser explicada por el nivel educacional más bajo, el rol de las usuarias como receptores de un sistema de salud determinado y por el sentido de autocrítica y de responsabilidad más alto de los profesionales $(5,11)$.

Las características socio-demográficas de los profesionales reflejan un rango amplio de edad y años de servicio, encontrando la mayor concentración de los profesionales en el nivel primario de atención, considerando que es a este nivel donde se detecta la posibilidad de detectar una LIE o un CC.

La Dimensión I (Funcionamiento del programa) en general fue bien evaluado por los dos grupos del estudio, lo que proyecta el grado de satisfacción global con la atención recibida y entregada, aspecto que ha sido descrito previamente como un determinante de la CA (9). Dentro de esta dimensión, cabe señalar que la comunicación establecida entre los profesionales y los usuarios de los servicios cobra un papel relevante a la hora de evaluar la CA (15). Varios son los investigadores que coinciden en que la comunicación que se entrega en los servicios de salud es ineficiente e inefectiva, que no se ajusta a las necesidades reales de las mujeres beneficiarias (5,16-18). Otros autores señalan que es indispensable al momento de evaluar la CA, considerar la cantidad y el tipo de información entregada (19), este aspecto de la primera dimensión, fue la más baja, donde usuarias como profesionales en los diferentes niveles de atención, la califican desfavorablemente, evaluación determinada, tal vez, por falta de trabajo en red en los diferentes niveles de atención, situación que fue verbalizada por algunos profesionales del estudio.

En la Dimensión II (Relación usuaria-profesional), es un aspecto relevante al momento de evaluar la CA $(20,21,22)$. Los resultados obtenidos concuerdan con la literatura, donde el buen trato resulta importante de ser evaluado $(7,23)$. Se observan diferencias entre ambos grupos de estudio, siendo nuevamente los profesionales los más críticos en su evaluación. Llama la atención dentro de esta dimensión, lo referido a si los profesionales se identifican por su nombre, donde las usuarias responden afirmativamente en un $54,7 \%$ versus un $11,9 \%$ de los profesionales. De la misma forma, las usuarias evalúan positivamente la opción que los profesionales dan para preguntar o manifestar inquietudes relacionadas a su enfermedad. Estos resultados obtenidos pueden deberse al sesgo en la información entregada por las participantes del estudio al no querer emitir un juicio real de su percepción por miedo a expresar su real experiencia. Al igual que los resultados encontrados en un estudio realizado en centros hospitalarios de nuestro país, la comunicación corresponde a un área concreta que necesita ser mejorada (5).

La Dimensión III (Infraestructura y recurso humano) en general, los resultados encontrados, son buenos, coincidiendo con otro estudio realizado en Chile (5). Otros autores reportan aspectos importantes, como los espacios físicos adecuados para la espera de la atención profesional como un elemento importante en la evaluación (22), elementos que en esta investigación, fueron bien evaluados por las usuarias, no así por los profesionales.

La Dimensión IV (Educación sobre la enfermedad) los resultados coinciden con los encontrados en la evaluación de un Programa de CC en Colombia, donde las mujeres señalan una buena educación tanto de la enfermedad como del tratamiento (24). En relación a este aspecto es importante educar e informar a la población objetivo de una forma adecuada y oportuna (16), orientada principalmente a dar a conocer la enfermedad, el pronóstico y los tratamientos existentes en la actualidad $(23,25)$, teniendo en cuenta que la mujer se encuentra vulnerable al momento de recibir el diagnóstico de cáncer, por lo que el apoyo brindado por parte del personal que la atiende adquiere un carácter fundamental (9). La educación permite a los usuarios ser más activos e informados en la toma de decisión relacionada a su enfermedad (26).

La Dimensión V (Recursos materiales), los aspectos relativos a la cantidad y calidad de los recursos materiales han sido evaluados como importantes en la evaluación de la CA desde la perspectiva de los beneficiarios de los servicios sanitarios (6), no encontrando en la literatura revisada evaluación de esta dimensión por parte de usuarios y prestadores de servicios de salud, que permita comparar nuestra realidad con otros sistemas o programas de salud. Los resultados reflejan un sentido muy crítico por parte de los profesionales, al ser esta la peor dimensión evaluada por ellos, situación que 
puede ser explicada por los conocimientos y las exigencias referentes a insumos y materiales que los profesionales requieren para entregar una atención eficiente para las usuarias.

La CA en salud es el resultado multifactorial de la satisfacción del proceso de cuidado de los usuarios de un programa o servicio. Asimismo los prestadores en salud tienen la responsabilidad y el compromiso ineludible de encontrar acciones concretas que permitan evaluar la CA entregada en los diferentes programas y servicios, con el fin de conocer las áreas deficientes e introducir mejoras en ellos.

Concordante con lo anterior la evaluación general del programa AUGE-CC que hacen tanto las usuarias como los profesionales, refleja la percepción de un buen funcionamiento del sistema, en donde la mayoría de los participantes de esta investigación recomendaría el programa. Del mismo modo identifica áreas de mejora emergentes a través de la comparación de percepciones, de usuarias y profesionales, como la dimensión relación usuaria profesional y la referente a recursos materiales, elementos claves en la evaluación de la CA.

Las diferencias entre la evaluación de usuarias y profesionales nos hace reflexionar respecto del nivel o estándar mínimo que las usuarias exigen en su atención. Muchas de ellas no conocen otros sistemas, porque no tienen acceso, por lo que el concepto de calidad de atención también se suscribe a la imposibilidad de ser atendida en otro lugar, lo que agrega cierto grado de resignación sesgando el patrón evaluativo.

\section{CONCLUSIÓN}

Se destaca la importancia que existan dentro en los servicios de salud, grupos de trabajos para identificar las necesidades, planificar estrategias tendientes a mejorar la CA, vigilar el cumplimiento de estándares, evaluar y coordinar los recursos que aseguren una óptima CA para las usuarias. Dichos equipos tienen un rol central en lo referente a dar una atención de calidad, sobre todo si hablamos de aquellos grupos más vulnerables del sistema de salud de nuestro país.

\section{BIBLIOGRAFÍA}

1. Gobierno de Chile, Ministerio de Salud. Los Objetivos Sanitarios para Chile 2000-2010. Hallado en: http:// epi.minsal.cl/epi/html/sdesalud/OS/OS2000_2010. $\mathrm{htm}$. Acceso el 28 de enero de 2011.

2. Ley № 19966. Régimen de Garantía Explícitas en Salud de Chile. Hallado en http://www.incasenjo.cl/ gtransparente/112010/Ley_19966.pd. Acceso el 28 de enero 2011.
3. Gobierno de Chile, Ministerio de Salud. AUGE 20052008. Implementación de garantías explícitas en salud. Hallado en: http://www.redsalud.gov.cl/portal/url/it em/9555f8a6fc41e874e04001011e017bf2.pdf. Acceso el 28 de enero 2011.

4. Bastías G, Valdivia G. Reforma de salud en chile; el plan AUGE o régimen de garantías explícitas en salud (GES). Su origen y evolución. Boletín Escuela de Medicina UC. Pontificia Universidad Católica de Chile 2007;32:51-8.

5. Riveros J, Berne C, Mujica J. Gestión y satisfacción en servicios de salud de Chile: Contraste entre las percepciones de los funcionarios y los usuarios. Rev Méd Chile 2010;138:630-8.

6. Ramírez T, Aguilar P, Nigenda G. Percepción de la calidad de la atención de los servicios de salud en México: perspectiva de los usuarios. Salud Pública México 1998;40:1-10.

7. Delgado-Gallego M, Vázquez M. Percepciones de usuarios y líderes comunitarios sobre su capacidad para influenciar en la calidad de los servicios de salud: un estudio de casos de Colombia y Brasil. Cad Saúde Pública 2009;25:169-78.

8. Reimann S, Strech D. The representation of patient experience and satisfaction in physician rating sites. A criteria-based analysis of English- and Germanlanguage sites. Reimann and Strech BMC Health Services Research [Publicación periódica en línea] 2010. [citada Feb 11], 10:3324. Hallado en: http://www.biomedcentral.com/1472-6963/10/32. Acceso el 4 de febrero 2011.

9. Urrutia MT, Poupin L, Padilla O. Construcción y validación de un instrumento para medir calidad de atención del programa AUGE Cáncer Cervicouterino. Rev Chil Obstet Ginecol 2010;75:396-404.

10. Hernandez ME, Ochando A, Mora J, Lorenzo S, López K. Satisfacción del paciente en una unidad de hemodiálisis: Objetivo de calidad asistencial en enfermería. Rev Soc Esp Esferm Nefrol 2006;8:90-6.

11. Mira J, Aranaz J, Lorenzo S, Rodríguez-Marín J, Moyano S. Evolución de la calidad percibida por los pacientes en dos hospitales públicos. Psicothema 2001;13:581-5.

12. Haas J, Cook E, Puoppolo A, Burstin H, Cleary $P$, Brennan $T$. Is the professional satisfaction of general internist associated with patient satisfaction? J Gen Intern Med 2000;15:122-8.

13. Landon B, Reschovsky J, Blumenthal D. Changes in career satisfaction among primary care and specialist physician 1997-2001. JAMA 2003;289:442-9

14. Urriola R. Algunos comentarios para evaluar el primer año del Plan Auge en el sistema público de salud. Rev Chil Salud Pública 2006;10:164-9.

15. Jayadevappa R, Schwart S, Chhatre S, Wein A, MaIkowicz B. Satisfaction with care: A measure of quality of care in prostate cancer patients. Med Decis Making 2010;30:234-45.

16. de Acevedo S, Antero Sousa M, Vieira N. Motivos que levan mulheres a nao retornarem para receber o resultado de examen Papanicolaou. Rev Latino-am Enfermagem 2006;14:503-9.

17. Herrera-Espiñeira C, Rodríguez del Aguila M, Rodrí- 
guez del Castillo M, Aguayo de Hoyos E, Quero A, Martínez C, et al. Evaluación de un programa de atención sanitaria con enfermera tutora en cirugía de traumatología en un hospital de Granada. Rev Esp Salud Pública 2004;82:69-80.

18. Tisheman C, Lundgren EL, Skald A, Tornberg S, Larsson BW. Quality of care from patient perspective in population-based cervical cancer screening. Acta Oncol 2002;41:253-61.

19. Lazcano-Ponce E, Moss S, Cruz-Valdez A, Alonso de Ruíz P, Martínez-León C, Casares-Queralt, et al. The positive experience of screening quality among users of a cervical cancer detection. Arch Med Res 2002; 33:186-92.

20. Awadalla A, Ohaeri J, Gholoum A, Khalid A, Hamad $H$, Jacob A. Factors associated with quality of life of outpatients with breast cancer and gynecologic cancers and their family caregivers: a controlled study. BMC Cancer 2007;19:102-15.

21. Bingham A, Bishop A, Coffey P, Winkler J, Bradley J, Dzuba I, et al. Factors affecting utilization of cervical cancer prevention services in low-resource setting. Salud Pública Mex 2003;45:408-16.
22. Ramírez T, Nájera P, Nigenda G. Percepción de la calidad de la atención de los servicios de salud en México: perspectiva de los usuarios. Salud Pública Mex 1998;4(1):3-12. Hallado en: http://www.scielosp.org/scielo.php?script=sci_arttext\&pid=S0036$36341998000100002 \&$ Ing=en\&nrm=iso. Acceso el 4 de febrero 2011.

23. Rolnick S, Lamerla J, Jackson J, Akkerman D, Compo R. Impact of a new cervical pap smear screening guideline on member perceptions and comfort levels. Prev Med 1999;28:530-4.

24. Noreña C, Tamayo L. Cáncer cérvicouterino: Análisis de la calidad de un programa. AQUICHAN 2010;10:52-68.

25. Farfán L, Barrón E, Jiméne $M$, Vásquez E, Ayala $S$. Factores socioculturales en mujeres que no acuden a detección oportuna de cáncer cervicouterino. Rev Enferm 2004;12:123-30.

26. Barber S, Gertler P. Empowering women to obtain high quality care: evidence from an evaluation of Mexico's conditional cash transfer programme. Health Policy Plan 2009;24:18-25. 